\title{
On the necessity of the assumptions used to prove Hardy-Littlewood and Riesz Rearrangement Inequalities
}

\author{
H. Hajaiej
}

\begin{abstract}
We prove that supermodularity is a necessary condition for the generalized HardyLittlewood and Riesz rearrangement inequalities. We also show the necessity of the monotonicity of the kernels involved in the Riesz-type integral.
\end{abstract}

Keywords and phrases: rearrangement, inequality, supermodular integrand .

AMS Subject Classification: 26D15 .

\section{Introduction}

Let $F: \mathbb{R}_{+}^{m} \rightarrow \mathbb{R}$ be a Borel measurable function. We say that $F$ is supermodular if:

$$
\begin{aligned}
& F\left(y+h e_{i}+k e_{j}\right)+F(y) \geq F\left(y+h e_{i}\right)+F\left(y+k e_{j}\right) \\
& \quad \text { for every } i, j \in\{1, \ldots, m\}, i \neq j, k, h>0
\end{aligned}
$$

where $y=\left(y_{1}, \ldots, y_{m}\right)$, and $e_{i}$ denotes the standard basis vector in $\mathbb{R}^{m}, i=1, \ldots, m$.

This condition has been first analysed in the context of integral inequalities by G. Lorentz [19.

Note that if $F$ is $C^{2}$, (1.1) is equivalent to

$$
\frac{\partial^{2} F}{\partial x_{i} \partial x_{j}}(x) \geq 0, \quad \text { for every } i, j \in\{1, \ldots, m\}, i \neq j .
$$

The supermodularity condition has been widely used in functional analysis. It was the main assumption to prove the generalized Hardy-Littlewood rearrangement inequality,

$$
\int_{X} F\left(u_{1}(x), \ldots, u_{m}(x)\right) d \mu(x) \leq \int_{X} F\left(u_{1}^{*}(x), \ldots, u_{m}^{*}(x)\right) d \mu(x),
$$

Here $u_{i}$ are nonnegative measurable functions that vanish at infinity and $u_{i}^{*}$ are their symmetric decreasing rearrangements. The integral is taken over $X=\mathbb{R}^{n}, \mathbb{H}^{n}$ or $\mathcal{S}^{n}$. (1.3) 
has been studied by many authors [17, 19, 11, 6, 7]. Its main applications are economics [11, chemistry 24], and nonlinear optics where the profile of stable electromagnetic waves traveling along a planar waveguide are given by the ground states of the energy functional

$$
E(u):=\frac{1}{2} \int_{\mathbb{R}}\left(u^{\prime 2}-G(|x|, u)\right) d x
$$

under the constraint $\|u\|_{2}=c>0$. A crucial step to prove that (1.4) admits an even ground state $u=u(x)$ which is decreasing for $x>0$, is (1.3), [15].

In [16], we showed that the ground state of the $m \times m$ elliptic system

$$
\text { (S) }\left\{\begin{array}{l}
\Delta u_{1}+\lambda_{1} u_{1}+g_{1}\left(u_{1}, \ldots, u_{m}\right)=0 \\
\ldots \\
\Delta u_{m}+\lambda_{m} u_{m}+g_{m}\left(u_{1}, \ldots, u_{m}\right)=0
\end{array},\right.
$$

where $g_{i}=\left(\partial G / \partial u_{i}\right),(i=1, \ldots, m)$, exists when $G$ is supermodular, and satisfies some further conditions.

Notice that supermodularity condition is equivalent to the cooperativity of $(\mathbf{S})$. As proved by W.C. Troy [24], the latter condition is also necessary for the existence of solutions of (S). In [10, G. Carlier viewed the generalized Hardy-Littlewood rearrangement inequality as an optimal transportation problem. He showed that the left-hand side of (1.3) achieves its maximum (i.e. the cost is minimized) under the supermodularity assumption. In this paper, we prove, among other things, that (1.1) is necessary for the inequality (1.3) to hold.

The generalized Riesz rearrangement inequality is

$$
\begin{aligned}
& \int \cdots \int F\left(u_{1}\left(x_{1}\right), \ldots, u_{m}\left(x_{m}\right)\right) \prod_{i<j} K_{i j}\left(d\left(x_{i}, x_{j}\right)\right) d x_{1} \cdots d x_{m} \\
\leq & \int \cdots \int F\left(u_{1}^{*}\left(x_{1}\right), \ldots, u_{m}^{*}\left(x_{m}\right)\right) \prod_{i<j} K_{i j}\left(d\left(x_{i}, x_{j}\right)\right) d x_{1} \cdots d x_{m},
\end{aligned}
$$

where $d$ denotes distance and the functions $K_{i j}$ are decreasing.

It is closely related to the Brunn-Minkowski inequality of convex geometry. Notice that the integral in (1.5) can represent a physical interaction potential.

The most relevant case is $K_{i j}\left(d\left(x_{i}, x_{j}\right)\right)=\omega_{i j}\left(\left|x_{i}-x_{j}\right|\right)$ where $\omega_{i j}$ are nonnegative nonincreasing functions. (1.5) is then equivalent to

$$
\begin{aligned}
& \int \cdots \int F\left(u_{1}\left(x_{1}\right), \ldots, u_{m}\left(x_{m}\right)\right) \omega_{i j}\left(\left|x_{i}-x_{j}\right|\right) d x_{1} \cdots d x_{m} \\
\leq & \int \cdots \int F\left(u_{1}^{*}\left(x_{1}\right), \ldots, u_{m}^{*}\left(x_{m}\right)\right) \omega_{i j}\left(\left|x_{i}-x_{j}\right|\right) d x_{1} \cdots d x_{m} .
\end{aligned}
$$

(1.5) and (1.6) have been studied in [22, 23, 3, 4, 5, 1, 20, 13, 21, 12, 25, 7]. All these results use the supermodularity of $F$. Among the numerous applications of (1.6), let us mention that it was extremely useful to prove the existence and uniqueness of the minimizing solution 
of Choquard's equation [18. In the special case that $F$ is a product and $m=2$, (1.6) hold on the standard spheres and hyperbolic spaces [2, 3] and it still contains the isoperimetric inequality as a limit. In this paper we will show (see Proposition 3.2 below) that (1.1) is necessary for (1.6) to hold. Notice that, in [7], cases of equality of (1.3) and (1.6) were established under the strict supermodularity condition, that is to say (1.1) with the strict inequality sign. We will also prove that such a condition is inescapable to establish cases of equality in (1.3) and (1.6) (see Remark 1 below).

\section{Notation and preliminaries}

We fix $n \in \mathbb{N}$. Let $\mu$ the Lebesgue measure on $\mathbb{R}^{n}$. If $x \in \mathbb{R}^{n}$ and $r>0$, let $B_{r}(x)=\left\{y \in \mathbb{R}^{n}\right.$ : $|y-x|<r\}$. For any set $M \subset \mathbb{R}^{n}$, let $\mathbf{1}_{M}$ denote its characteristic function. By $\mathcal{M}$ we denote the set of all measurable functions on $\mathbb{R}^{n}$. For a Borel measurable function $F: \mathbb{R}_{+}^{m} \rightarrow \mathbb{R}$, nonincreasing functions $w_{i j}: \mathbb{R}_{+} \rightarrow \mathbb{R}_{+}$and nonnegative $u_{i} \in \mathcal{M}$, we study the following generalized Hardy-Littlewood type functional,

$$
I\left(u_{1}, \ldots, u_{m}\right)=\int_{\mathbb{R}^{n}} F\left(u_{1}(x), \ldots, u_{m}(x)\right) d x,
$$

and the generalized Riesz type functional.

$$
J\left(u_{1}, \ldots, u_{m}\right)=\int_{\mathbb{R}^{n}} \cdots \int_{\mathbb{R}^{n}} F\left(u_{1}\left(x_{1}\right), \ldots, u_{m}\left(x_{m}\right)\right) \omega_{i j}\left(\left|x_{i}-x_{j}\right|\right) d x_{1} \cdots d x_{m} .
$$

If $f \in \mathcal{F}$, let $f^{*}$ denote its Schwarz symmetrization which is the unique lower continuous function which is radially symmetric, radially nonincreasing and such that $\mu\{a<f \leq b\}=$ $\mu\left\{a<f^{*} \leq b\right\}$ for all numbers inf $f<a<b$ (see [1]). Notice that if $f=\mathbf{1}_{M}$, where $M$ is Lebesgue measurable with finite measure, then $f^{*}=\mathbf{1}_{B_{R}(0)}$ where $R$ is chosen such that $\mu\left(B_{R}(0)\right)=\mu(M)$. Accordingly, a function $u \in \mathcal{F}$ is called Schwarz symmetric if it is radial and radially decreasing. We say that it is strictly Schwarz symmetric if it is radial and strictly radially decreasing.

\section{Results}

Proposition 3.1 ( Necessity of the supermodularity condition in the generalized HardyLittlewood inequality )

Let $F:\left(\mathbb{R}_{+}\right)^{m} \rightarrow \mathbb{R}$ be a Borel measurable function which vanishes on hyperplanes, that is, $F\left(y_{1}, \ldots, y_{m}\right)=0$ if $y_{k}=0$ for some $k \in\{1, \ldots, m\}$. If

$$
\int_{\mathbb{R}^{n}} F\left(u_{1}(x), \ldots, u_{m}(x)\right) d x \leq \int_{\mathbb{R}^{n}} F\left(u_{1}^{*}(x), \ldots, u_{m}^{*}(x)\right) d x,
$$

for any $\left(u_{1}, \ldots, u_{m}\right) \in \mathcal{F}^{m}$ then $F$ satisfies (1.1). 
Proof: Suppose that (1.1) is not true. Then there exist $i, j \in\{1, \ldots, m\}, i \neq j, 0 \leq a<b$, $0 \leq c<d$ and $m-2$ nonnegative numbers $\alpha_{l},(l \in\{1, \ldots, m\} \backslash\{i, j\})$, such that:

$$
\begin{aligned}
& F\left(\alpha_{1}, \ldots, a, \ldots, d, \ldots, \alpha_{m-2}\right)+F\left(\alpha_{1}, \ldots, b, \ldots, c, \ldots, \alpha_{m-2}\right) \\
>\quad & F\left(\alpha_{1}, \ldots, b, \ldots, d, \ldots, \alpha_{m-2}\right)+F\left(\alpha_{1}, \ldots, a, \ldots, c, \ldots, \alpha_{m-2}\right) .
\end{aligned}
$$

Now let $E$ and $F$ be two measurable sets such that $E \cap F=\emptyset, \mu(F)=\mu(E)<\infty$, and $u_{i}=a \mathbf{1}_{E}+b \mathbf{1}_{F}, u_{j}=c \mathbf{1}_{F}+d \mathbf{1}_{E}$, and $u_{l}=\alpha_{l}\left(\mathbf{1}_{F}+\mathbf{1}_{E}\right)$ for $l \in\{1, \ldots, m\} \backslash\{i, j\}$. Then $u_{i}^{*}=b \mathbf{1}_{B_{r}(0)}+a \mathbf{1}_{A}$, where $r$ is chosen such that $\mu\left(B_{r}(0)\right)=\mu(F)=\mu(E)$, and $A$ is the annulus $B_{r^{\prime}} \backslash \overline{B_{r}}$ and $\mu(A)=\mu(F)=\mu(E)$. It follows that $u_{j}^{*}=d \mathbf{1}_{B_{r}(0)}+c \mathbf{1}_{A}, u_{l}^{*}=\alpha_{l} \mathbf{1}_{B_{r^{\prime}}(0)}$, and

$$
\begin{aligned}
& \int F\left(u_{1}(x), \ldots, u_{n}(x)\right) d x \\
= & {\left[F\left(\alpha_{1}, \ldots, a, \ldots, d, \ldots, \alpha_{m-2}\right)+F\left(\alpha_{1}, \ldots, b, \ldots, c, \ldots, \alpha_{m-2}\right)\right] \mu(E), } \\
& \int F\left(u_{1}^{*}(x), \ldots, u_{n}^{*}(x)\right) d x \\
= & {\left[F\left(\alpha_{1}, \ldots, b, \ldots, d, \ldots, \alpha_{m-2}\right)+F\left(\alpha_{1}, \ldots, a, \ldots, c, \ldots, \alpha_{m-2}\right)\right] \mu(E) . }
\end{aligned}
$$

Hence we have in view of (3.1),

$$
\int F\left(u_{1}(x), \ldots, u_{n}(x)\right) d x>\int F\left(u_{1}^{*}(x), \ldots, u_{n}^{*}(x)\right) d x
$$

a contradiction.

Remark 1: Following the same approach we can easily conclude that the strict supermodularity assumption, that is, (1.1) with strict inequality sign, is necessary to establish cases of equality. In [7, (1.3) was proven under the supermodularity assumption (1.1) and an integrability condition, and cases of equality were established assuming (1.1) with strict inequality sign.

Proposition 3.2: ( Necessity of supermodularity assumption in Riesz-type integral ) Let $j:(0,+\infty) \rightarrow \mathbb{R}$ be a function which is nonincreasing and not identically equal to zero, and such that

$$
\lim _{r \rightarrow+\infty} r^{n-1} j(r)=0
$$

Suppose that $\Psi:[0,+\infty) \times[0,+\infty) \rightarrow \mathbb{R}$ is a Borel measurable function satisfying

$$
\Psi(u, 0)=\Psi(0, v)=0 \quad \forall u \geq 0, v \geq 0 .
$$

Finally assume that for all nonnegative functions $f, g \in L^{\infty}\left(\mathbb{R}^{n}\right)$ with compact support there holds

$$
\int_{\mathbb{R}^{n}} \int_{\mathbb{R}^{n}} \Psi(f(x), g(y)) j(|x-y|) d x d y \leq \int_{\mathbb{R}^{n}} \int_{\mathbb{R}^{n}} \Psi\left(f^{*}(x), g^{*}(y)\right) j(|x-y|) d x d y .
$$


Then $\Psi$ is supermodular, that is,

$$
\Psi\left(a+a^{\prime}, b+b^{\prime}\right)-\Psi\left(a+a^{\prime}, b\right)-\Psi\left(a, b+b^{\prime}\right)+\Psi(a, b) \geq 0 \quad \forall a, a^{\prime}, b, b^{\prime} \in[0,+\infty) .
$$

Proof: In view of (3.2), and since $j$ is non-increasing and nontrivial, $j$ is non-negative, too, and there exist positive numbers $\varepsilon$ and $t_{0}$ with $t_{0}>2 \varepsilon$ and such that

$$
j(t)<j(s) \quad \text { whenever } 0 \leq s \leq \varepsilon \text { and }\left|t-t_{0}\right| \leq \varepsilon .
$$

We fix $z \in \mathbb{R}^{n}$ with $|z|=t_{0}$, and $\rho>t_{0}+\varepsilon$.

Now let $a, a^{\prime}, b, b^{\prime} \in[0,+\infty)$. Then, if $f=a \mathbf{1}_{B_{R}(0)}+a^{\prime} \mathbf{1}_{B_{\varepsilon}(0)}$, and $g=b \mathbf{1}_{B_{\rho}(0)}+b^{\prime} \mathbf{1}_{B_{\varepsilon}(z)}$, where $R>\varepsilon$, we have that $f=f^{*}$, and $g^{*}=b \mathbf{1}_{B_{\rho}(0)}+b^{\prime} \mathbf{1}_{B_{\varepsilon}(0)}$. Using (3.3), a short computation shows that (3.4) is equivalent to

$$
\begin{aligned}
0 \leq & \left\{\Psi\left(a+a^{\prime}, b+b^{\prime}\right)-\Psi\left(a+a^{\prime}, b\right)-\Psi\left(a, b+b^{\prime}\right)+\Psi(a, b)\right\} \\
& \cdot \int_{B_{\varepsilon}(0)}\left[\int_{B_{\varepsilon}(0)} j(|x-y|) d y-\int_{B_{\varepsilon}(z)} j(|x-y|) d y\right] d x \\
+ & \left(\Psi\left(a, b+b^{\prime}\right)-\Psi(a, b)\right) \int_{B_{R}(0)}\left[\int_{B_{\varepsilon}(0)} j(|x-y|) d y-\int_{B_{\varepsilon}(z)} j(|x-y|) d y\right] d x
\end{aligned}
$$

Set

$$
H(x):=\int_{B_{\varepsilon}(0)} j(|x-y|) d y, \quad x \in \mathbb{R}^{n} .
$$

Since $j$ is nonnegative and nonincreasing, $H$ is nonnegative, radial and radially nonincreasing, that is we can write $H(x)=: h(|x|)$, with $h$ nonincreasing. Since $j$ satisfies (3.2), we also have $\lim _{t \rightarrow \infty} t^{n-1} h(t)=0$. Setting

$$
I(t):=\int_{B_{t}(0)} H(x) d x-\int_{B_{t}(z)} H(x) d x, \quad(t>0),
$$

it follows that $I$ is nonnegative, and moreover, (3.6) implies that

$$
I(\varepsilon)>0 .
$$

Finally, if $t>t_{0}$, then

$$
I(t) \leq \int_{B_{t+t_{0}}(0) \backslash B_{t-t_{0}}(0)} H(x) d x=n \omega_{n} \int_{t-t_{0}}^{t+t_{0}} s^{n-1} h(s) d s,
$$

where $\omega_{n}$ denotes the volume of the unit ball in $\mathbb{R}^{n}$. Hence

$$
\lim _{t \rightarrow \infty} I(t)=0
$$

Now inequality (3.7) becomes

$$
\begin{aligned}
0 & \leq\left\{\Psi\left(a+a^{\prime}, b+b^{\prime}\right)-\Psi\left(a+a^{\prime}, b\right)-\Psi\left(a, b+b^{\prime}\right)+\Psi(a, b)\right\} I(\varepsilon) \\
& +\left(\Psi\left(a, b+b^{\prime}\right)-\Psi(a, b)\right) I(R) .
\end{aligned}
$$


Sending $R \rightarrow+\infty$, we obtain

$$
0 \leq\left\{\Psi\left(a+a^{\prime}, b+b^{\prime}\right)-\Psi\left(a+a^{\prime}, b\right)-\Psi\left(a, b+b^{\prime}\right)+\Psi(a, b)\right\} I(\varepsilon),
$$

and (3.5) follows from (3.8).

Remark 2: Proposition 3.2 tells us in particular that $\Psi$ is supermodular if (3.4) holds for $j=\mathbf{1}_{B_{R}(0)}$, with some $R>0$.

Proposition 3.3. Let $\Psi:[0,+\infty) \times[0,+\infty)$ be nontrivial, and satisfies (3.5) and (3.3), and let $h \in C\left(\mathbb{R}^{n}\right)$. Assume that there holds

$$
\int_{\mathbb{R}^{n}} \int_{\mathbb{R}^{n}} \Psi(f(x), g(y)) h(x-y) d x d y \leq \int_{\mathbb{R}^{n}} \int_{\mathbb{R}^{n}} \Psi\left(f^{*}(x), g^{*}(y)\right) h(x-y) d x d y
$$

for all bounded nonnegative functions $f$ and $g$ with compact support. Then $h$ is radial and radially nonincreasing, that is, it can be written as $j(|x|)=h(x),\left(x \in \mathbb{R}^{n} \backslash\{0\}\right)$, where $j:(0,+\infty) \rightarrow \mathbb{R}$ is a nonincreasing function.

Proof : By choosing $a=b=0$ in (3.5) and taking into account (3.3), we have that $\Psi$ is nonnegative, and since $\Psi$ is nontrivial, there are numbers $a>0, b>0$ such that $\Psi(a, b)>0$. Let $z_{1}, z_{2} \in \mathbb{R}^{n}$ with $0<\left|z_{1}\right|<\left|z_{2}\right|$, and set $R:=\left(\left|z_{1}\right|+\left|z_{2}\right|\right) / 2$. Let $f=a\left(\mathbf{1}_{B_{R}(0)}+\mathbf{1}_{B_{\varepsilon}\left(z_{2}\right)}-\right.$ $\left.\mathbf{1}_{B_{\varepsilon}\left(z_{1}\right)}\right)$, and $g=b \mathbf{1}_{B_{\varepsilon}(0)}$, where $0<\varepsilon<\left(\left|z_{2}\right|-\left|z_{1}\right|\right) / 2$. Then $g=g^{*}$ and $f^{*}=a \mathbf{1}_{B_{R}(0)}$. Hence (3.11) gives

$$
0 \leq \Psi(a, b)\left\{\int_{B_{\varepsilon}\left(z_{1}\right)} \int_{B_{\varepsilon}(0)} h(x-y) d x d y-\int_{B_{\varepsilon}\left(z_{2}\right)} \int_{B_{\varepsilon}(0)} h(x-y) d x d y\right\} .
$$

Sending $\varepsilon \rightarrow 0$ and taking into account $\Psi(a, b)>0$ we find that $h\left(z_{1}\right) \geq h\left(z_{2}\right)$. Since $h$ is continuous, this implies $h(x) \geq h(y)$ iff $|x| \leq|y|$. Hence $h$ is radial and radially nonincreasing as claimed.

Acknowledgement: The author is very grateful to F. Brock for his kind invitation to visit AUB in June 2008 and June 2009, and for his precious help.

\section{References}

[1] F. Almgren, E.H. Lieb, Symmetric decreasing rearrangement is sometimes continuous. Journal of the A.M.S. 2 (1989), 683-773.

[2] A. Baernstein II, B.A. Taylor, Spherical rearrangements, subharmonic functions and *-functions in n-space. Duke Math. J. 43 (1976), 245-268.

[3] W. Beckner, Sobolev inequalities, the Poisson semigroup and the analysis on the sphere $\mathcal{S}^{n}$. Proc. A.M.S. 89 (1992), 4816-4819. 
[4] W. Beckner, Sharp Sobolev inequalities on the sphere and the Moser-Trudinger Inequality. Ann. Math. (2) 138 (1993), 213-242.

[5] H.J. Brascamp, E.H. Lieb, M.J. Luttinger, A general rearrangement inequality for multiple integrals. J. Funct. Anal. 17 (1974), 227-237.

[6] F. Brock, A general rearrangement inequality a la Hardy-Littlewood. J. Inequ. Appl. 5 (2000), 309-320.

[7] A. Burchard, H. Hajaiej, Rearrangement inequalities for functionals with monotone integrands. Journal of Functional Analysis 233 (2006), 561-582.

[8] A. Burchard, M. Schmuckenschläger, Comparison theorems for exit times. Geom. Funct. Anal. 11 (2001), 651-692.

[9] G.R. Burton, Vortex-rings of prescribed impulse. Proc. Cambridge Philos. Soc. (3) 134 (2003), 515-528.

[10] G. Carlier, On a class of multidimensional optimal transportation problems. J. Convex Anal. 10 (2003), 517-529.

[11] J.A. Crowe, J.A. Zweibel, P.C. Rosenbloom, Rearrangement of functions. J. Funct. Anal. 66 (1986), 432-438.

[12] C. Draghici, A general rearrangement inequality. Proc. Amer. math. Soc. 133 (2005), $735-743$.

[13] R. Friedberg, J.M. Luttinger, (a) Rearrangement inequalities for periodic functions, (b) A new rearrangement inequality for multiple integrals. Arch. Rat. Mech. Anal. 61 (1976), 35-44, and 45-64.

[14] H. Hajaiej, Balls are the maximizers of the Riesz-type functionals with supermodular integrands. to appear in: Annali de Matematica Pura ed Applicata.

[15] H. Hajaiej, C.A. Stuart, Existence and nonexistence of Schwarz symmetric ground states for eigenvalue problems. Ann. Mat. Pura Appl. (4) 184 (2005), no. 3, 297-314.

[16] H. Hajaiej, Symmetric ground states solutions of m-coupled nonlinear Schrdinger equations. Nonlinear Analysis: Methods, Theory and Applications 71, no.2 (2009).

[17] G.E. Hardy, J.E. Littlewood, G. Polya, Inequalities. Cambridge Univ. Press, London and N.Y, 1st edn. 1934, 2nd edn. 1952.

[18] E.H. Lieb, Existence and uniqueness of the minimizing solution of Choquard's nonlinear equation. Studies in Applied Mathematics 57 (1977), 93-105. 
[19] G.G. Lorentz, An inequality for rearrangements. Amer. Math. Monthly 60 (1953), 176179.

[20] J.M. Luttinger, Generalized isoperimetric inequalities I,II,III. J. Math. Physics 14 (1973), 586-593, 1444-1447, 1448-1450.

[21] C. Morpurgo, Sharp inequalities for functional integrals and traces of conformally invariant operators. Duke Math. J. 114 (2002), 447-553.

[22] F. Riesz, Sur une inegalite integrale. J. London Math. Soc. 5 (1930), 162-168.

[23] S.L. Sobolev, On a theorem in functional analysis. Math. Sb. (N.S.) 4 (1938), 471-497; AMS Transl.(2) 34 (1963), 39-68.

[24] W. Troy, Symmetry properties in systems of semilinear elliptic equations. J. Differential Equations 42 (1981), no. 3, 400-413.

[25] A. Zygmund, On an integral inequality. J. London Math. Soc. 8 (1933), 175-178. 